\title{
Improvement of control at network node based on fuzzy controls
}

\author{
Nguyen Kim Quoc ${ }^{1, *}$, Vuong Xuan $\mathrm{Chi}^{1}$ and Nguyen Van Thanh ${ }^{1}$ \\ ${ }^{1}$ Nguyen Tat Thanh University, Vietnam
}

\section{Abstract}

\begin{abstract}
One of the main tasks of controlling communications at the network node is managing the queues, maintaining a reasonable probability of proactive packet removal to minimize congestion, while ensuring the quality of traffic flows and equity in the relationship between traffic flows, when the dynamic state of the network changes. However, current queue management mechanisms still need to be improved to both simplify implementation and improve intelligence in maintaining the average queue length. Therefore, the paper focuses on improving the queue management mechanism at the network node, on the basis of applying the achievements of the soft computing field to supplement the adaptability, the ability to learn, the ability to intelligent decision making for queue management system at the network node.
\end{abstract}

Keywords: Congestion Control, Active Queue Management, Fuzzy Logic

Received on 26 October 2019, accepted on 02 November 2019, published on 29 November 2019

Copyright (C) 2019 Nguyen Kim Quoc et al., licensed to EAI. This is an open access article distributed under the terms of the Creative Commons Attribution licence (http://creativecommons.org/licenses/by/3.0/), which permits unlimited use, distribution and reproduction in any medium so long as the original work is properly cited.

doi: 10.4108/eai.13-7-2018.163406

\section{Introduction}

Active queue management works at network nodes to control the number of packets in the network node's queues, by actively discarding packets until the queue is full or reporting congestion while the network is in "embryonic" period of congestion to regulate network traffic. Stabilizing the length of the queue will make some performance parameters of TCP / IP network such as packet loss rate, line usage efficiency, average delay and variation of latency fluctuation within a range. reasonable. This will not only cause overload for network devices, but also ensure no congestion on the network, and facilitate the provision and maintenance of the best network service quality [1][35] [36].

There are currently three approaches to solving the problem of active queue management, including: Queue management based on queue length (typically the RED mechanism) [13], management queue management based on incoming packet traffic - also known as loading (represented by BLUE mechanism) [23] and queue management based on a combination of queue length and packet traffic to (typically REM mechanism) [30]. In recent years, in order to improve the performance of active queue management mechanisms, in addition to the three typical ones mentioned above, many other mechanisms have been published. These works revolve around improving the RED, BLUE and REM mechanisms [9].

By analyzing AQM mechanisms using fuzzy logic, these improvement projects of AQM mechanisms based on queue length and speed of incoming traffic, surrounding the modification of RED and REM. In these mechanisms, FEM [7] and FUZREM [38] are the two mechanisms presented in details, clearly and offering the best results. Therefore, we will analyze and assess these two mechanisms to clarify the operations of AQM mechanisms using fuzzy logic. Generally, these mechanisms apply fuzzy control using Mamdani fuzzy system of triangular functions, owning deductive fixed lawful system, input variables are the difference between the instantaneous queue length with referential queue length, taken on two consecutive times and output variables are the probability of packet marking. This makes control mechanisms rough and the adaptability low. Thus, we propose solutions to build adaptive fuzzy controller (temporarily called AFC) combined with present active queue management mechanisms to improve the performance of the active queue management mechanisms [4][7][8].

So, the opening of the paper presents the mathematic base of fuzzy logic, next is a survey to assess the active queue management mechanisms using existing fuzzy logic. Since then, we propose the adaptive fuzzy control model to improve the active queue management mechanism in order to

*N. K. Quoc: nkquoc@ntt.edu.vn 
overcome some limitations in existing mechanisms. Based on the theoretical model, we deploy to install simulation, develop FLREM and FLRED innovative mechanisms. In particular, the FLRED mechanism improves the RED mechanism based on the queue length, the FLREM mechanism improves the REM mechanism based on the combination of the queue length with the speed of incoming traffic. The next of the paper is the evaluation on simulation of mechanisms proposed compared to traditional mechanisms and mechanisms using fuzzy control. The simulation results of AQM mechanisms show the performance of traditional mechanisms increased when applying fuzzy control, and this factor is enhanced when using the adaptive fuzzy controller - AFC for mechanisms.

\section{Related Works}

\subsection{Operation of RED mechanism}

Sally Floyd and Van Jacobson proposed mechanism RED [13] for the early detection of congestion, RED is one of the mechanisms used to control the congestion at the network node by checking the average length when the packet queue and make a decision to mark or discard the packet to the probability increases as the average length of the queue exceeds a set threshold.

When packets arrive at network nodes, RED active queue management mechanism calculate the average queue length by the formula: $\hat{k}=(1-\omega) * \hat{k}+\omega * k$, with $\omega$ is weighted queue to control the volatility of average size $(0 \leq \omega \leq 1)$ and $k$ is the current queue length

Then RED compare the average queue length with two thresholds minimum $\min _{t h}$ and maximum $\max _{t h}$. If the average queue length is less than $\mathrm{min}_{\text {th }}$ do not have any packages marked (or probability marked by 0 ). When the average queue length greater than $\max _{t h}$, all incoming packets are marked (or probability marked by 1 ) and in fact this package is removed. When the average queue length is between $\min _{t h}$ and $\max _{t h}$ each incoming packet is marked with a probability $p_{a} \in$ $\left[0, \max _{p}\right]$, is defined by the formula (1) and (2) follows:

$$
\begin{gathered}
p_{b}=\max _{p} \frac{\hat{k}-\min _{t h}}{\max _{t h}-\min _{t h}} \\
p_{a}=\frac{p_{b}}{1-\text { count }^{*} p_{b}}
\end{gathered}
$$

\subsection{Operation of REM mechanism}

The first idea of REM is stable input rate and link capacity of the queue, regardless of the number of users sharing links [30]. Each output queue of router installed REM mechanism and maintains a variable called 'price'. Price as a factor in evaluating congestion. Price is updated periodically or asynchronously, based on the asymmetry of the asymmetric load and the asymmetry queue size. The load is unworthy of the difference between the speed of the flow of data into and existing capacity of the link. Unworthiness of the queue size is the difference between the required target queue size with queue size of the router.
This price increase if the total weight of unworthiness is positive, and decreases in the opposite case. Total weight is positive when one input exceeds the binding capacity or there are too many packages in the queue backlog should be cleared, and negative in the opposite case. As the number of users increases, the load is not uniform among the queue size increases, boosting the price and therefore the probability of congestion marked. This will send a stronger signal congestion to the source, then the source of reduced growth. When downloading the source is too small, will not sync negative, and marked probability reduce and enhance the of load power. Until unworthiness is towards to zero, high efficiency and negligible decrease, so the delay in equilibrium. The queue is deleted in equilibrium if the queue set to 0 .

REM clear control through updates its price, this is the first character of REM. Exactly, queue size $l$, price $p_{l}(t)$ in stages $t$ is updated by the formula following [1][20]:

$$
p_{l}(t+1)=\left[p_{l}(t)+\gamma\left(\alpha_{l}\left(b_{l}(t)-b_{l}^{*}\right)+x_{l}(t)-c_{l}(t)\right)\right]^{+}
$$

Where, $\gamma>0$ and $\alpha_{l}>0$ constants are small and $[z]^{+}=\max \{z, 0\}$. Here, $b_{l}(t)$ the average queue size of the queue $l$ in time $\mathrm{t}$ and $b_{t}^{*} \geq 0$ the target queue length, $x(t)$ average download speeds of queue $l$ at the time of $t$, and $c_{l}(t)$ allowed bandwidth of queue $l$ at the time of $t$. The difference in load is $x_{l}(t)-c_{l}(t)$ and the queue size $b_{l}(t)-b_{l}^{*}$. The constant $\alpha_{l}$ can be set up by each individual queue and are updated according to the performance and latency in each queue. The constant $\gamma$ REM control response changes depending on network conditions. Parameters price will increase if the speed deviation of the load and queue size is positive and the price will decrease in the opposite case. At equilibrium, stability and $\mathrm{p}$ is calculated using the formula:

$$
\alpha_{l}\left(b_{l}(t)-b_{l}^{*}\right)+x_{l}(t)-c_{l}(t)=0
$$

This can be kept when loading the input speed equal to the speed of processing in routers $\left(x_{l}=c_{l}\right)$ and the number of packets in the queue with the desired queue size $b_{l}=b_{l}^{*}$

REM's second idea is to use a total of price links along the path of the packet, this total reflects signs of congestion on the path, the total price to be embedded into the terminal to mark probability. Forms exponentially increase the probability of marking is very important in a large network. At last, the probability of marking a packet passes through multiple congested link from source to destination depends on the probabilities associated marks at all links in the path. When and only when the associated component marking probability is exponential in its price link. This led to the arrival terminal will be marked probability increases exponentially with the total price of all links in congested link in its path. This total is a true measure of congestion in the path of the packets. Since it is embedded in the terminal to mark probability, it can be easily estimated from the sources of its own packets are marked, and is used to design adaptation loads of it.

Suppose that a packet passes through the links $l=1,2, \ldots, L$ have price $p_{l}(t)$ at time $t$, the marking probability $m_{l}(t)$ queue 
$l$ at time $t$ is:

$$
m_{l}(t)=1-\phi^{-p_{l}(t)}
$$

Where, $\phi>1$ is constant and marking probability at the end of the packet is [18][19][22]:

$$
1-\prod_{l=1}^{L}\left(1-m_{l}(t)\right)=1-\phi^{-\sum_{l} p_{l}(t)}
$$

Probability high marks when large $\operatorname{sum} \sum_{l} p_{l}(t)$. When the marking probability at the link $m_{l}(t)$ small, should $p_{l}(t)$ small. So, the probability is marked at the end by the above formula is proportional to the sum of the $p_{l}(t)$ along the way and mark probability at end approximately: $\left(\log _{e} \phi\right) \sum_{l} p_{l}(t)$.

\subsection{Operation of FEM mechanism}

C. Chrysostomou et al. Published a positive FEM queue management mechanism by improving the RED mechanism based on fuzzy arguments [7]. The goal of the FEM mechanism is to use fuzzy control to adjust the network node's queue length within a predefined domain range, in order to achieve the reference queue length (TQL: Target Queue Length), maintaining the level use high queue and low average latency.

FEM uses Mamdani fuzzy system whose input variable is the instantaneous queue length deviation from the reference queue length at two consecutive times e (kT) and e (kT-T) and the output is determined. packet markup rate $\mathrm{p}(\mathrm{kT})$. FEM mechanism blurs the input value by a function in the form of a triangle with 5 value domains representing the fuzzy calculation of input e (kT), e (kT-T). Figure 1. allows fuzzy representation of the input e (kT), e (kT-T).

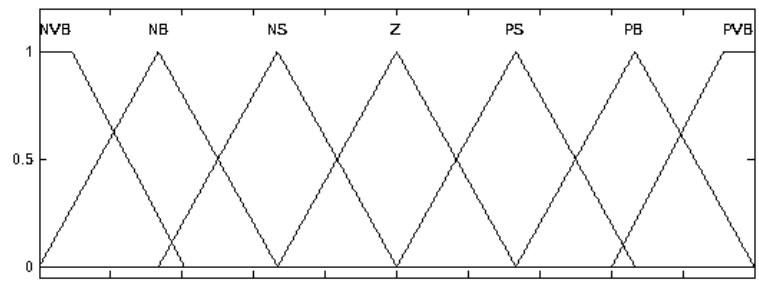

Figure 1. The membership function of FEM's input language variables

The values of the linguistic variable chosen to denote cases that occur for two inputs e (kT) and e (kT-T) are: NVB (Negative Very Big); NB (Negative Big); NS (Negative Small); Z (Zero); PS (Positive Small); PB (Positive Big Big); PVB (Positive Very Big).

Similarly, the FEM mechanism fades the output value to the probability of marking or discarding the $\mathrm{p}(\mathrm{kT})$ package as follows: Z (Zero); T (Tiny); VS (Very Small); S (Small; B (Big); VB (Very Big); H (Huge), blurred by a function of a triangle with 7 value domains Figure 2. shows the fuzzy function of $\mathrm{p}(\mathrm{t})$ at the output

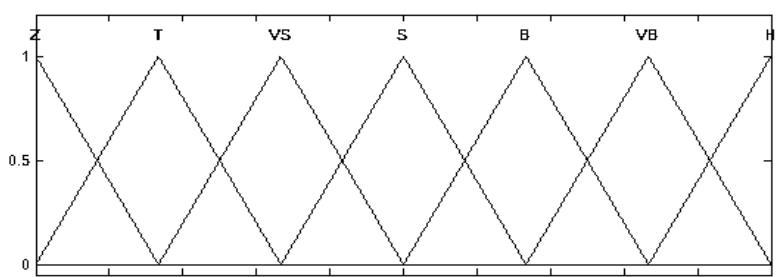

Figure 2. The membership function of FEM's output language variables

According to the evaluation, FUZREM works very well when stabilizing the queue length according to the desired value and reducing packet latency in the queue. However, like FEM, the FUZREM mechanism has a number of shortcomings that affect its operation. For example, FUZREM's fuzzy parameters are not smooth due to the use of a triangular function, FUZREM's fuzzy parameter sets cannot automatically update and FUZREM does not have a mechanism to adjust the target value itself as well as an adaptive mechanism for probability of marking packets at output.

Because each input variable has 7 value domains, FEM's rule set is composed of 49 rules $(7 \times 7)$, which are described in Table 1.

Table 1. System of law inference FEM

\begin{tabular}{|c|c|c|c|c|c|c|c|c|}
\hline \multirow{2}{*}{\multicolumn{2}{|c|}{$p(k T)$}} & \multicolumn{7}{|c|}{$e(k T-T)$} \\
\cline { 2 - 10 } & NVB & NB & NS & Z & PS & PB & PVB \\
\hline \multirow{5}{*}{$e(k T)$} & NVB & H & H & H & H & H & H & H \\
\cline { 2 - 9 } & NB & B & B & B & VB & VB & H & H \\
\cline { 2 - 9 } & NS & T & VS & S & S & B & VB & VB \\
\cline { 2 - 9 } & $\mathrm{Z}$ & $\mathrm{Z}$ & $\mathrm{Z}$ & $\mathrm{Z}$ & $\mathrm{T}$ & $\mathrm{VS}$ & $\mathrm{S}$ & $\mathrm{B}$ \\
\cline { 2 - 9 } & $\mathrm{PS}$ & $\mathrm{Z}$ & $\mathrm{Z}$ & $\mathrm{Z}$ & $\mathrm{Z}$ & $\mathrm{T}$ & $\mathrm{T}$ & $\mathrm{VS}$ \\
\cline { 2 - 9 } & $\mathrm{PB}$ & $\mathrm{Z}$ & $\mathrm{Z}$ & $\mathrm{Z}$ & $\mathrm{Z}$ & $\mathrm{Z}$ & $\mathrm{Z}$ & $\mathrm{T}$ \\
\cline { 2 - 9 } & $\mathrm{PVB}$ & $\mathrm{Z}$ & $\mathrm{Z}$ & $\mathrm{Z}$ & $\mathrm{Z}$ & $\mathrm{Z}$ & $\mathrm{Z}$ & $\mathrm{Z}$ \\
\hline
\end{tabular}

Each cell in the table represents a deductive rule, such as the top left cell representing the rule: if (e (kT) is NVB) and (e (kT-T) is NVB) then p (kT) is H. That is, if the instant queue length deviation from the reference queue length is "very large negative" and the deviation of the queue length from the previous cycle compared to the reference queue length is "very large negative" "The probability of marking the packet as" very large".

\subsection{Operation of FUZREM mechanism}

X. Changbiao et al. Proposed an active FUZREM queue management mechanism based on the improvement of the REM mechanism with the fuzzy controller [38]. FUZREM uses the price function (variable pl) to measure the instantaneous congestion of the REM mechanism and determines the deviation of the queue length from the desired queue length (variable perr) to calculate the probability of packet marking.

The input variable $\mathrm{pl}$ of the FUZREM fuzzy controller is 
fuzzyized by a function of a triangle with 7 value ranges as follows: Z (Zero); T (Too Small); VS (Very Small); S (Small); B (Big); VB (Very Big); H (Huge). Similarly, the perr input variable of FUZREM fuzzy controller is dimmed by a function of triangular shape with 5 values and trapezoidal function with 2 values set at the beginning and the end.

The shape of the perr membership functions is similar to Figure 1, the values of the linguistic variable chosen to denote cases that occur for perr are: NB (Negative Big); NM (Negative Medium); NS (Negative Small); Z (Zero); PS (Positive Small); PM (Positive Medium); PB (Positive Big). FUZREM's fuzzy controller output is the probability of marking a dropped packet that is fuzzy in a triangle function with 7 different values in the segment $[0,1]$. Based on input and output variables, FUZREM fuzzy controller is built with 49 rules in Table 2. as follows

Table 2. System of law inference FUZREM

\begin{tabular}{|c|c|c|c|c|c|c|c|c|}
\hline \multicolumn{2}{|c|}{ drop $(k T)$} & \multicolumn{7}{|c|}{$\operatorname{Perr}(k T)$} \\
\cline { 2 - 9 } \multicolumn{1}{|c|}{} & NB & NM & NS & Z & PS & PM & PB \\
\hline \multirow{5}{*}{$p l(k T)$} & $\mathrm{Z}$ & $\mathrm{Z}$ & $\mathrm{Z}$ & $\mathrm{Z}$ & $\mathrm{Z}$ & $\mathrm{Z}$ & $\mathrm{Z}$ & $\mathrm{Z}$ \\
\cline { 2 - 9 } & $\mathrm{T}$ & $\mathrm{Z}$ & $\mathrm{Z}$ & $\mathrm{Z}$ & $\mathrm{Z}$ & $\mathrm{Z}$ & $\mathrm{Z}$ & $\mathrm{T}$ \\
\cline { 2 - 9 } & $\mathrm{VS}$ & $\mathrm{Z}$ & $\mathrm{Z}$ & $\mathrm{Z}$ & $\mathrm{T}$ & $\mathrm{T}$ & $\mathrm{T}$ & $\mathrm{VS}$ \\
\cline { 2 - 9 } & $\mathrm{S}$ & $\mathrm{Z}$ & $\mathrm{Z}$ & $\mathrm{T}$ & $\mathrm{T}$ & $\mathrm{VS}$ & $\mathrm{S}$ & $\mathrm{B}$ \\
\cline { 2 - 9 } & $\mathrm{B}$ & $\mathrm{VS}$ & $\mathrm{S}$ & $\mathrm{S}$ & $\mathrm{S}$ & $\mathrm{B}$ & $\mathrm{VB}$ & $\mathrm{VB}$ \\
\cline { 2 - 9 } & $\mathrm{VB}$ & $\mathrm{B}$ & $\mathrm{B}$ & $\mathrm{VB}$ & $\mathrm{VB}$ & $\mathrm{H}$ & $\mathrm{H}$ & $\mathrm{H}$ \\
\cline { 2 - 9 } & $\mathrm{H}$ & $\mathrm{H}$ & $\mathrm{H}$ & $\mathrm{H}$ & $\mathrm{H}$ & $\mathrm{H}$ & $\mathrm{H}$ & $\mathrm{H}$ \\
\hline
\end{tabular}

\section{Fuzzy controller}

\subsection{Mathematical representation of the fuzzy approximation}

At the MISO fuzzy systems are nonlinear mapping from input vectors $x=\left[x_{1}, \ldots, \mathrm{x}_{n}\right]^{T} \in \mathfrak{R}^{n}$ to output $\mathrm{y} \in \mathfrak{R}$ (Figure 3 ). In the theory of fuzzy sets and fuzzy logic ([4], [8]), allows dimming chemical inputs using fuzzy operators chemical transfer function data clearly the basis of fuzzy sets and fuzzy rules with the assumption including: $p$ fuzzy rules are represented as a set of fuzzy descriptive (fuzzy Implications) after [8][17][34]:

$$
\mathfrak{R}_{i}:\left(A_{J_{1 i}}^{1} \cap A_{J_{2 i}}^{2} \cap \ldots A_{J_{n i}}^{n}\right) \Rightarrow B_{k i}
$$

with $i=1 . . p$, notation $A \Rightarrow B$ or just fuzzy describe for conditions statements If A Then B and $A_{j}^{i}, B_{k}$ is the fuzzy set is defined as follows:

$$
\begin{gathered}
A_{j}^{i}=\left\{\left(x_{i}, \mu_{A_{j}^{i}}\left(x_{i}\right)\right): \mathrm{x}_{i} \in \mathfrak{R}\right\} \\
B_{k}=\left\{\left(y, \mu_{B_{k}}(y)\right): y \in \mathfrak{R}\right\}
\end{gathered}
$$

with $\mu_{A_{j}^{i}}, \mu_{B_{k}} \in[0,1]$ respectively, the membership functions of $j$ and $k$ inputs $x_{i}$ and output $y$.
The basic problem of fuzzy systems in fuzzy inference mechanism (fuzzy inference) and defuzzification methods (defuzzification) used to calculate the output of fuzzy system clearly specify when the input given on the basis of fuzzy rules have known. This fuzzy inference mechanism is built on the success of inference rules. To calculate the the first clause in the formula (7) we can use any t-norm does [30] as the smallest t-norm $T_{M I N}(a, b)=\min \{a, b\}$, algebraic integrated $T_{P A N D}(a, b)=a b$, Łukasiewicz function $T_{\text {LAND }}(a, b)$ $=\max \{a+b-1,0\}$, In case of using smallest t-norm $T_{M I N}$ then fuzzy describe (15) can be written as Decac integrated $\left(A_{J_{1 i}}^{1} \times A_{J_{2 i}}^{2} \times \ldots \times A_{J_{n i}}^{n}\right) \Rightarrow B_{k i}$ the first clause in the formula (7) calculated as follows:

$$
\mu_{A_{J_{1 i}}^{1} \times A_{J_{2 i}}^{2} \times \ldots \times A_{J_{n i}}^{n}}(x)=\min \left\{\mu_{A_{J_{1 i}}^{1}}\left(x_{1}\right), \mu_{A_{J_{2 i}}^{2}}\left(x_{2}\right), \ldots, \mu_{A_{J_{n i}}^{n}}\left(x_{n}\right)\right\}
$$

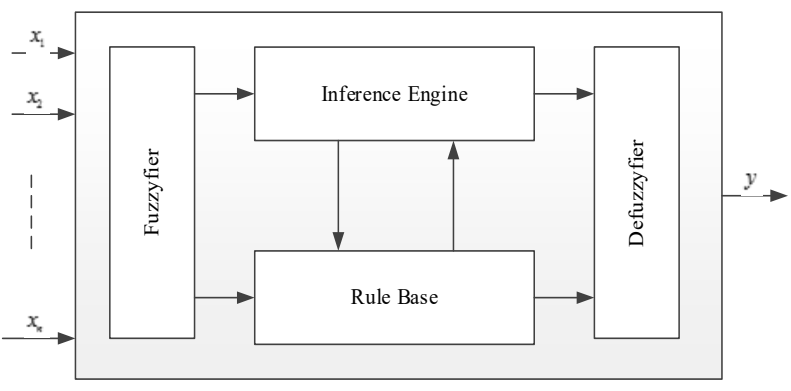

Figure 3. MISO fuzzy control system

To calculate the fuzzy description of each rule or the output of each rule can be used to describe the fuzzy operator ( $t$-norm or t-conorm). Some fuzzy operator describe common include: Zadeh $x \Rightarrow y=\max \{1-x, \min \{x, y\}\}$, Lukasiewicz $x \Rightarrow y$ $=\min \{1,1-x+y\}$, Mamdani $x \Rightarrow y=\min \{x, y\}$ và Larsen $x \Rightarrow y=x y$. Where the operator using fuzzy Mamdani describes the output of the $i^{\text {th }}$ rule $\left(R_{i}\right)$, denoted as $C_{i}=\left\{\left(y, \mu_{C_{i}}(x, y)\right): x \in \mathfrak{R}^{n}, \mathrm{y} \in \mathfrak{R}\right\}$ calculated as follows:

$$
\begin{aligned}
& \mu_{C_{i}}(x, y)=\mu_{A_{J_{1 i}}^{1} \times A_{J_{2 i}}^{2} \times \ldots \times A_{J_{n i}}^{n}}(x, y) \\
& =\min \left\{\mu_{A_{J_{1 i}}^{1} \times A_{J_{2 i}}^{2} \times \ldots \times A_{J_{n i}}^{n}}(x), \mu_{B_{k i}}(y)\right\}
\end{aligned}
$$

Finally, to calculate the output of fuzzy system can be used as a defuzzification method of defuzzification focal point COG (Center Of Gravity) after:

$$
y=F(x, \theta)=\frac{\sum_{i=1}^{p} C_{i} \int_{z} \mu_{C_{i}}(x, z) d z}{\sum_{i=1}^{p} \int_{z} \mu_{C_{i}}(x, z) d z}
$$

with $c_{i}$ is the center of $\mu_{B_{k i}}(y)$ for to $i^{\text {th }}$ rule. Normally we choose $\mu_{B_{k i}}(y)$ symmetry across a vertical axis through the peak to $c_{i}$ is the midpoint of $\mu_{B_{k i}}(y)$

The equation (19) is a model of Mamdani fuzzy system with COG defuzzification method and is used as the fuzzy 
approximation $F(\mathbf{x}, \boldsymbol{\theta})$ with $\theta=\left[c_{1}, \ldots, \mathbf{c}_{p}\right]^{T}$.

\subsection{Fuzzy logic controller}

Fuzzy logic controllers, like expert systems, can be used to model human experiences and human decision making behaviors. In FLC the input-output relationship is expressed by using a set of linguistic rules or relational expressions. The FLC basically consists of four important parts including a fuzzifier, a defuzzifier, an inference engine and a rule base. As in many fuzzy control applications, the input data are usually crisp, so a fuzzification is necessary to convert the input crisp data into a suitable set of linguistic value that is needed in inference engine. Singleton fuzzifier is the general fuzzification method used to map the crisp input to a singleton fuzzy set. In the rule base of a FLC, a set of fuzzy control rules, which characterize the dynamic behavior of system, are defined. The inference engine is used to form inferences and draw conclusions from the fuzzy control rules. Figure 3 shows the fuzzy logic controller architecture. The output of inference engine is sent to defuzzification unit. Defuzzification is a mapping from a space of fuzzy control actions into a space of crisp control actions [15][19][25].

1) Layer of Inputs: language input variable is the variable that represents the main factors affecting the operation mechanism of REM.

2) Layer of Fuzzifier: this layer performs the function of tissues in the value of the variable input, will pass each of the input values to set the corresponding language.

3) Layer of Rules: this layer contains the basis for inference rules. Code base is a set of fuzzy rules of the form IF-THEN, for $n$ variables in $x_{1, . .}, x_{n}$, the $y$ variable, fuzzy rules of the form $\mathrm{R}: \mathrm{IF}\left(x_{1}\right.$ is $\left.A_{1}\right) \wedge . . \wedge\left(x_{i}\right.$ is $\left.A_{i}\right) \wedge \ldots \wedge\left(x_{n}\right.$ is $\left.A_{n}\right)$ THEN $y$ is $B$. Where $A_{i}$ and $B$ are fuzzy sets of linguistic variables $x_{1}, x_{2}, \ldots$, $x_{n}$ and the outcome variable $y$.

4) Layer of Inference Engine: this layer performs the function of the total results of the nodes in the rule layer to send, through the permit OR (Max).

5) Layer of Defuzzifier: functional class implements defuzzification to obtain results, the results are calculated probability of packet marking optimization under the current state of the network. The output $f(x)$ of this fuzzy controller with singleton fuzzifier, inference engine and center-average defuzzifier can be calculated as:

$$
f(x)=\frac{\sum_{j=1}^{k} y_{0}^{j} \cdot \prod_{i=1}^{n} \mu_{i}^{j}\left(x_{i}\right)}{\sum_{j=1}^{k} \prod_{i=1}^{n} \mu_{i}^{j}\left(x_{i}\right)}
$$

Where $\mathrm{y}_{0}$ is the center value of the output fuzzy set $b$, $\mu(x)$ is the membership function for fuzzy sets. In our proposed model we use two input variables to fuzzy controller which present the congestion measures for the packet loss, queue length and the output will be the drop probability value.

\subsection{Mamdani fuzzy system}

Mamdani fuzzy controller (also known as estimates Control) used control method of Mamdani is first fuzzy control method. It is used in cases where the condition clause and the clause conclusions are fuzzy values, the following general form [4][7][9]:

$$
\begin{aligned}
& R_{i}: \operatorname{IF}\left(x_{1} \text { is } \mathrm{A}_{1}^{j}\right) \wedge \ldots \\
& \wedge\left(x_{m} \text { is } \mathrm{A}_{m}^{j}\right) \operatorname{THEN}\left(y_{1} \text { is } B_{1}^{j}\right), \ldots,\left(y_{n} \text { is } B_{n}^{j}\right)
\end{aligned}
$$

Where $\mathrm{m}$ is the number of the input signal, $\mathrm{n}$ is the number of output signal, $j=1 \ldots k$, where $\mathrm{k}$ is the number of control rules. Conclusion of Mamdani fuzzy control methods are fuzzy clauses.

\subsection{Sugeno fuzzy system}

Sugeno fuzzy model published by Takagi, Sugeno, Kang desire to develop a systematic approach to establish the fuzzy rules from the data set into place. Basic fuzzy Sugeno model has the form [4][7][9]::

$$
\begin{gathered}
I F\left(x_{1} \text { is } A_{1}^{j}\right) A N D \ldots A N D\left(x_{n} \text { is } A_{n}^{j}\right) \\
\text { THEN } y=f_{j}=p_{0}^{j}+\sum_{i=1}^{n} p_{i}^{j} x_{i}
\end{gathered}
$$

With $A_{i}$ the fuzzy conditions and $y=f_{j}$ is clearly a function of the conclusions. Usually $f_{j}$ polynomial of the input variables $x_{i}$ or any function as long as the appropriate describe the output of the fuzzy system in the domain defined by the premise of the rule.

\subsection{Problems exist $A Q M$ using fuzzy logic}

In general, the mechanism of fuzzy control applications using Mamdani fuzzy system with the function of triangular shape, with the input variable is the difference between the instantaneous queue length with reference queue length, taken on two consecutive time and the output variable is the probability of packet marking. However, these mechanisms also exist a number of the following:

- First, the use of triangular membership functions to calculate simple, but it makes the controller is not smooth [36]. To overcome this drawback, we offer solutions in the form of a bell-shaped function used to represent values of fuzzy variables in the input and output of the fuzzy controller.

- Second, the value of the variable probability of marking packets at the output of the fuzzy controller is shown the qualitative factors of the people participating in the control system, this value has to be some standard mechanism of the interval $[0,1]$. However, in the FEM, the coefficients are adjusted by hand, there should be self-correcting mechanism in the output coefficient for adjusting the value of the packet marking probability of the system quantitatively, to be valid interest in accordance with the status of the network.

- Third, the reference queue length $\left(q_{\text {ref }}\right)$ used in the mechanism is set fixed value, usually the threshold is $80 \%$ of the capacity of the system (such as buffer size). However, there are strategies to build a model to change the reference value in accordance with the state of the network. For example, when the road is clear, then set the value to low- 
latency queue in small, whereas the high reference value set to limit the loss.

- Fourth, the parameters of the fuzzy controller (number of functions, the value of the function, the number of laws, rules weights) were fixed since the design, should not be changed to suit the situation network.

- Thursday, the major cause for the AQM mechanism using fuzzy controller performance is not high due to this mechanism using Mamdani fuzzy system for their fuzzy controller. According to the researchers in the field of automatic control, the Mamdani fuzzy system does not work effectively with Sugeno fuzzy systems, especially in the control system such MISO [7] [10].

To overcome this limitation of the mechanism, we propose to model AFC using fuzzy Sugeno with a bell-shaped function of the adjustment mechanism and target values self-control mechanism control output probabilities. AFC fuzzy controller built into the rest of the article.

\section{Proposed adaptive fuzzy controller}

\subsection{Building adaptive fuzzy controller}

The adaptive fuzzy control (AFC) model with the central processor is a fuzzy controller, there are structural adjustment parameters and parameters in place, so that the output of the model most similar models. Figure 4. represents a model adaptation:

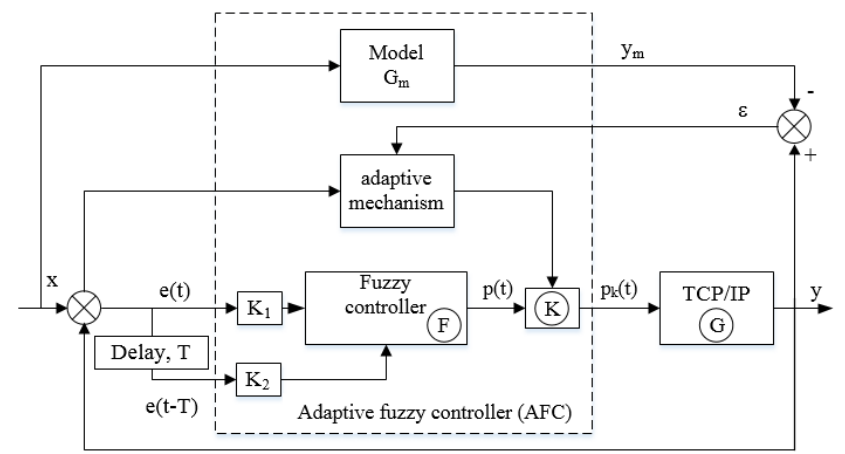

Figure 4. Improved model AQM mechanism by AFC

The AFC model is described as follows:

- Based on the input state that $G_{m}$ determine reference output $y_{m}$ (queue length, bandwidth or a combination of both factors) for the network. The value is set $y_{m}$ depending resource capacity of the system and the status of the network and is set $y_{m}=\alpha y_{\max }$.

- Closed-loop control system, the input value $e(t), e(t-T)$ is the deviation of output $y$ compared with the reference value $y_{m}$ will be standardized by $K_{l}$ and $K_{2}$ the previous coefficients going into the fuzzy controller.

- After the input data in a standardized, data will be sent to the fuzzy controller. Here, fuzzy control system components: fuzzy sets, the rule, the inference engine and the defuzzification as described in Section 3.1 will operate and value package marking probability $p(t)$.
- Based on $\varepsilon$ allowed deviation between the reference value $y_{m}$ with output value $y$ and the actual deviation $e(t)$ of the system where the structure is adapted to adjust the output of the $\mathrm{K}$ for appropriate fuzzy control. So the probability value marked packets at the output of the AFC fuzzy controller is adapted $p_{k}(t)=K \cdot p(t)$.

\subsection{Fuzzy control of AFC}

Fuzzy controller for the AFC is based on Sugeno fuzzy system is presented in Section 4.1 with the following components.

\subsection{Factor input}

The value of the input variable $e(t)$ và $e(t-T)$ should be standardized domain of appropriate values for the fuzzy and later trained by the sigmoid function. Therefore, all values must be normalized in the interval $[-1,1]$. Therefore, the input coefficients $\mathrm{K}_{1}, \mathrm{~K}_{2}$ is defined, so that $-1 \leq e(t) . K_{1} \leq 1$; $-1 \leq e(t-T) . K_{2} \leq 1$ and is calculated using the formula $(2: 22)$

$$
K_{i}=\left\{\begin{array}{ll}
1 / y_{m} & \text { when } y<y_{m} \\
1 /\left(y_{m}-y\right) & \text { when } y \geq y_{m}
\end{array} ; i=1,2\right.
$$

In that $y_{\max }$ is the maximum value of the model, $y_{m}$ the reference value functions. When $y<y_{m}$ (output shown below the reference level) is $0<e(t) . K_{i}=\left(y_{m}-y\right) / y_{m}<$ $1(i=1,2 ; t=k T, k T-T)$.

Conversely, when $y \geq y_{m}$ (shown on the reference output) is $\quad-1<e(t) . K_{i}=\left(y_{m}-y\right) /\left(y_{\max }-y_{m}\right) \leq 0 \quad(i=$ $1,2 ; t=k T, k T-T)$.

\section{Fuzzy input variable}

To determine the inference rule system, we need to describe the linguistic variables to be loaded into the fuzzy controller to describe the object in the best way. The variable input language is set to "false queue", "false flow rate to" or "false prices" in two consecutive time is $e(t)$ và $e(t-T)$.

To simplify the computation, the usual functions of triangular, trapezoidal used for language processing. However, this is not the function of the normal distribution function to the control values are not slippery. So we used the bell-shaped membership functions for the fuzzy input variables. In the fuzzy controller, we use the method with the functions of symmetric and equidistant, the sum of the value of an input language is always equal to 1 .

The value of the selected language to denote the case of two input $e(t)$ and $e(t-T)$ corresponding to two consecutive times and fuzzy membership functions by the bell-shaped with seven domain values (numerical values of FEM domain and FUZREM) is: $\{\mathrm{NB}, \mathrm{NM}, \mathrm{NS}, \mathrm{ZE}, \mathrm{PS}, \mathrm{PM}, \mathrm{PB}\}$. Meaning of dismissal following values: Negative Big (NB), Negative Medium (NM), Negative Small (NS), Zero (ZE), Small Positive (PS), Positive Medium (PM, Positive Big (PB). The function of the input linguistic variables $e(t)$ and 
$e(t-T)$ are shown in Figure 5 as follows:

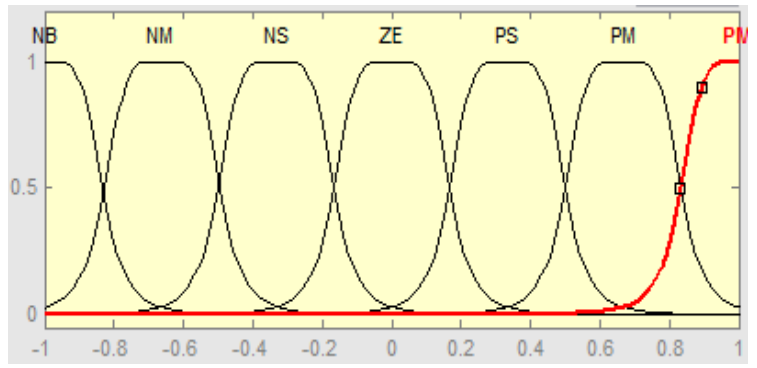

Figure 5. Membership functions of the input variables

\section{Fuzzy output variable}

Similarly, we define the language value for the output variable is the probability of marking / remove packages as follows $p(t)$ : Zero (Z), Tiny (T), Very Small (VS), Small (S), Big (B), Very Big (VB), Huge $(\mathrm{H})$. The value $p(t)$ is fuzzy singleton with values representing the 7 levels in the interval $[0,1]$ as follows:

$$
\begin{aligned}
& p(t)=\{\mathrm{H}, \mathrm{VB}, \mathrm{B}, \mathrm{S}, \mathrm{VS}, \mathrm{T}, \mathrm{Z}\} \\
& =\{0.000,0.167,0.333,0.500,0.667,0.834,1.000\}
\end{aligned}
$$

\section{Construction fuzzy inference rules}

The set of fuzzy rules is designed in detail by selecting the simplest model MISO (avoid the exponential increase of the set of fuzzy rules and thus reduce the complexity of the control system) based on two objectives: A comprehensive system of rule means that all the circumstances of the behavior of the system must be considered. This means that the combination of the input variables change will lead to a corresponding output. The rules of the fuzzy controller is based on a formula (14). Do we have two inputs, each input value for the domain seven for variable languages so there will be 49 fuzzy inference system.

To facilitate the construction and building the fuzzy rules, the index for the domain associated value of the variable input language $K_{1} \cdot e(t), K_{2} \cdot e(t-T)$ is $\{\mathrm{NB}, \mathrm{NM}, \mathrm{NS}, \mathrm{ZE}, \mathrm{PS}, \mathrm{PM}, \mathrm{PB}\}=$ $\left\{\mu(e)_{-3}, \mu(e)_{-2}, \mu(e)_{-1}, \mu(e)_{0}, \mu(e)_{1}, \mu(e)_{2}, \mu(e)_{3}\right\} \quad$ and output linguistic variables $p(t)$ is $\{\mathrm{H}, \mathrm{VB}, \mathrm{B}, \mathrm{S}, \mathrm{VS}, \mathrm{T}, \mathrm{Z}\}=$ $\left\{\mu(p)_{-3}, \mu(p)_{-2}, \mu(p)_{-1}, \mu(p)_{0}, \mu(p)_{1}, \mu(p)_{2}, \mu(p)_{3}\right\}$.

Fuzzy system is built according to the following general form:

IF $K_{1} \cdot e(t)=\mu(e(t))_{i}$ and $K_{2} \cdot e(t-T)=\mu(e(t-T))_{j}$ THEN $p(t)=\mu(p(t))_{k}$

Where, $i, j=\overline{-3,3}$, and $k=f(i, j)=i+j$, if $i+j>3$ then $f(i, j)=3$ and if $i+j<-3$ then $f(i, j)=-3$.

Example: If $K_{1} \cdot e(t)=-2(\mathrm{NB})$ and $K_{2} \cdot e(t-T)=$ -1 (NS) then $p(t)=-3(H)$.

If the reference is incorrect large negative (NB) and false negative reference before small (NS), then the probability of marking / remove packages are very large $(\mathrm{H})$.

Based on this general fuzzy systems, fuzzy systems with the rules in the following Table 3.
Table 3. Index systems of fuzzy rules

\begin{tabular}{|c|c|r|r|r|r|r|r|r|}
\hline \multirow{2}{*}{$p(t)$} & \multicolumn{7}{|c|}{$K_{2} \cdot e(t-T)$} \\
\cline { 2 - 8 } & -3 & -2 & -1 & 0 & 1 & 2 & 3 \\
\hline \multirow{5}{*}{$K_{1} \cdot e(t)$} & 3 & 0 & 1 & 2 & 3 & 3 & 3 & 3 \\
\cline { 2 - 8 } & 2 & -1 & 0 & 1 & 2 & 3 & 3 & 3 \\
\cline { 2 - 8 } & 1 & -2 & -1 & 0 & 1 & 2 & 3 & 3 \\
\cline { 2 - 8 } & 0 & -3 & -2 & -1 & 0 & 1 & 2 & 3 \\
\cline { 2 - 8 } & -1 & -3 & -3 & -2 & -1 & 0 & 1 & 2 \\
\cline { 2 - 8 } & -2 & -3 & -3 & -3 & -2 & -1 & 0 & 1 \\
\cline { 2 - 8 } & -3 & -3 & -3 & -3 & -3 & -2 & -1 & 0 \\
\hline
\end{tabular}

Based on the values domain index of the linguistic variables for input and output in Table 3 , build rule system as Table 4.

Table 4. Fuzzy rule system

\begin{tabular}{|c|l|r|r|r|r|r|r|r|}
\hline \multicolumn{2}{|c|}{$p(t)$} & \multicolumn{7}{|c|}{$K_{2} \cdot e(t-T)$} \\
\cline { 2 - 10 } & & $\mathrm{NB}$ & $\mathrm{NM}$ & $\mathrm{NS}$ & $\mathrm{ZE}$ & $\mathrm{PS}$ & $\mathrm{PM}$ & $\mathrm{PB}$ \\
\hline \multirow{5}{*}{$K_{1} \cdot e(t)$} & $\mathrm{PB}$ & $\mathrm{VS}$ & $\mathrm{T}$ & $\mathrm{Z}$ & $\mathrm{Z}$ & $\mathrm{Z}$ & $\mathrm{Z}$ & $\mathrm{Z}$ \\
\cline { 2 - 9 } & $\mathrm{PM}$ & $\mathrm{S}$ & $\mathrm{VS}$ & $\mathrm{T}$ & $\mathrm{Z}$ & $\mathrm{Z}$ & $\mathrm{Z}$ & $\mathrm{Z}$ \\
\cline { 2 - 9 } & $\mathrm{PS}$ & $\mathrm{B}$ & $\mathrm{S}$ & $\mathrm{VS}$ & $\mathrm{T}$ & $\mathrm{Z}$ & $\mathrm{Z}$ & $\mathrm{Z}$ \\
\cline { 2 - 10 } & $\mathrm{ZE}$ & $\mathrm{VB}$ & $\mathrm{B}$ & $\mathrm{S}$ & $\mathrm{VS}$ & $\mathrm{T}$ & $\mathrm{Z}$ & $\mathrm{Z}$ \\
\cline { 2 - 9 } & $\mathrm{NS}$ & $\mathrm{H}$ & $\mathrm{VB}$ & $\mathrm{B}$ & $\mathrm{S}$ & $\mathrm{VS}$ & $\mathrm{T}$ & $\mathrm{Z}$ \\
\cline { 2 - 9 } & $\mathrm{NM}$ & $\mathrm{H}$ & $\mathrm{H}$ & $\mathrm{VB}$ & $\mathrm{B}$ & $\mathrm{S}$ & $\mathrm{VS}$ & $\mathrm{T}$ \\
\cline { 2 - 10 } & $\mathrm{NB}$ & $\mathrm{H}$ & $\mathrm{H}$ & $\mathrm{H}$ & $\mathrm{VB}$ & $\mathrm{B}$ & $\mathrm{S}$ & $\mathrm{VS}$ \\
\hline
\end{tabular}

\section{Surfaces inference}

The surface of this inference is the most simplified representation of the fuzzy controller. The output is an interpolation made two inputs. Reconciling surface nonlinear control and the language rule is used, will provide hints about the operation of fuzzy control method using surface inference probability bring better marks than other approaches, so close to human reasoning and the nonlinear available. Figure 6 illustrates the curved surface of the inference rule system is described in Table 4.

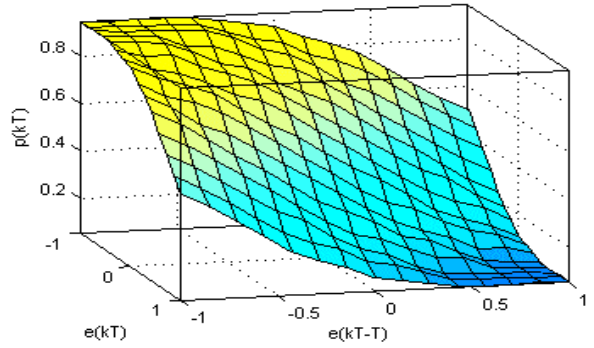

Figure 6. Surfaces inference of AFC

From fuzzy inference on the surface, we can easily calculate the probability of the area marked equilibrium, where the error on the queue length is almost zero. On the other hand, the active rule by increased ability to mark packets in areas beyond the equilibrium point where 
congestion began to be established.

\section{Fuzzy out variable}

To control the decision was final, the output fuzzy sets must be converted into an output value clearly through the defuzzifier allowed. Here, the fuzzy method are central domain of fuzzy output after combined and are shown in formula:

$$
\begin{aligned}
& \text { (a) } p_{k}=\frac{\int y \mu_{c}(y) d y}{\int \mu_{c}(y) d y} \\
& \text { (b) } p_{k}=\frac{\sum_{j=1}^{m} y_{j} \mu_{c}\left(y_{j}\right)}{\sum_{j=1}^{m} \mu_{c}\left(y_{j}\right)}
\end{aligned}
$$

In particular $\mu_{c}(y)=\max \left(\mu_{1}(y), \mu_{2}(y), \ldots, \mu_{N}(y)\right)$ is the dependence of $y$ in fuzzy combined $C$ and $N$ is the number of inference rules. Limits of integration allows the formula (17a) corresponds to space medicine and the probability value marking / removal which contains $y$. To reduce the computational process we discretize space $Y$ into $m$ values and core values obtained as formula (17b)

\subsection{Building sample model}

The design goal of the AQM algorithm is to determine the limits of queue fluctuations, fluctuations in flow rate to or fluctuate delays, queue need to adjust the speed of incoming traffic or at a suitable level. This leads to the need to have a reference input is fixed reference queue length, or flux from the sample model fit $G_{m}$. Based on maximum capacity $y_{\max }$ (queue length, link bandwidth) in sample model to establish reference values $y_{m}$ corresponding.

This parameter can be set so as to obtain the average delay variation and low latency. For the purpose of keeping instantaneous queue length at a fixed rate, control the flux does not change. Fuzzy control algorithm allows tighter control, avoiding losses and the volatility is not acceptable around the reference point. This contrasts with the traditional AQM algorithms, control the macroscopic behavior of the queue length and packet flow to, they often result in slow response and fluctuations in queue length / store instantaneous load, leading to large latency variability.

\subsection{Determine the input and output variables}

To work properly, the system should set the range of values for the input variables to suit the system. The range of values can be determined by testing with different input values for the control system to determine the extent of variation of error in a sampling interval. We choose the value of the error $e(t)$ and the rate of change of error based on the value added at the time of the error $e(t-T)$ as input on the fuzzy controller. That is, the input is the deviation of the instantaneous output value $(y)$ compared with the reference output value $\left(y_{m}\right)$ at two consecutive time is $(t)$ and $(t-T)$. The expression $e(t)=y_{m}-y(t)$ determines the current time and errors $e(t-T)=y_{m}-y(t-T)$ was determined time error after a delay time $\mathrm{T}$ (in cycles taken previous sample). Similarly, we need to consider the probability of selection marker / discard the packet $p(t)$ as the output of the fuzzy controller.

\subsection{Adaptive mechanism}

Adaptive mechanism to adjust the output of the $\mathrm{K}$ values that mark the appropriate probabilities. When the output value exceeds the reference value, it is necessary to increase $\mathrm{K}$ to the probability marks and vice versa. Reduced gradient method applied to the adaptive correction coefficient K output of the fuzzy controller. The process of adjustment is performed according to main structures are referred to as adaptive fuzzy controller according to sample model.

Objects TCP/ IP has transfer function G, sample model with transfer function $G_{m}$, the fuzzy controller with transfer function $F$. Need to find a rule correction coefficient $\mathrm{K}$ so that the difference between the actual value $\mathrm{y}$ and the reference value $y_{m}$ toward $0(\varepsilon \rightarrow 0)$. Based on the model in Figure 4, the controller output ratio are defined as follows::

$$
\begin{aligned}
& y=\frac{K F G}{1+K F G} y_{m} \\
& \frac{\partial \varepsilon}{\partial K}=-\frac{\partial y}{\partial K}=-\frac{K F G}{(1+K F G)^{2}} \cdot \frac{y_{m}}{K}=-\frac{K F G}{1+K F G} \cdot \frac{\varepsilon}{K}
\end{aligned}
$$

with $\frac{y_{m}}{1+K F G}=\varepsilon, y \rightarrow y_{m}$ then $\mathrm{KFG} /(1+\mathrm{KFG}) \approx 1$.

Therefore, we have: $\frac{\partial \varepsilon}{\partial K}=-\frac{\varepsilon}{K}$ and $K$ values were calculated by the formula:

$$
K(t+1)=K(t)-\varepsilon\left(y_{m}-y(t)\right)
$$

Based on the formula $(2: 26)$, the output value exceeds the reference level $\left(y(t)>y_{m}\right)$, the increased value of the coefficient $K$ to increase the value of the packet marking probability. Conversely, when the output values below the reference $\left(y(t)<y_{m}\right)$, the reduced value of the coefficient $K$ to reduce the value of the packet marking probability. Upon reaching steady state, the output value of the reference value $\left(y(t)=y_{m}\right)$ is kept constant $K$ value.

\section{Improve RED mechanisms by AFC}

According to the analysis and evaluation of the traditional AQM mechanisms, as well as analysis and evaluation of the AQM mechanism that uses fuzzy logic, then the improvements AQM mechanisms based on the way long queues around the amendment RED. Therefore, in the construction of improved mechanisms AQM mechanism by AFC adaptive fuzzy control, we continue to choose the improvements represent RED mechanisms based on queue length. AFC Model for FLRED mechanism is based on AFC general model for improving AQM mechanisms is presented in Section 4.1 as follows 


\subsection{Fuzzy input variables for FLRED}

Using a general improvement model as shown in Figure 4 with $\mathrm{T}$ is the sampling period. With $Q e(t), Q e(t-T)$ is misleading the immediate queue length compared to the reference queue TQL in the cycle of sampling and sampling in the previous cycle. This deviation is normalized values in the interval $[-1,1]$ before putting them into the fuzzy controller by $K_{1}$ and $K_{2}$ to the coefficient determined by the formula (15). The value of the selected language to denote the case of two input $K_{1}$. $Q e(t), K_{2} \cdot Q e(t-T)$ was constructed as in Section 4.3. Context describes how to express the value of linguistic variables as follows:

- Statement "deviation queues are positive big (PB)" describes situations where the queue immediate below it than the reference value TQL.

- Statement "deviation queues are positive big and deviation queue before is negative small" describes a situation where the queue while the lower than the reference value TQL and it tends to go away in the direction down compared queue with reference values.

\subsection{Fuzzy output variables for FLRED}

Output linguistic variables for the fuzzy controller of FLRED mechanism is packet marking probability $p(t)$. The value of linguistic variable $p(t)$ represents the level of packet marking and built seven-level degree is described in Section 4.3.2. Context expression language variables as follows: statements "probabilistic packet marking Zero (Z)" described the situation is not marked packets discarded, packets are put into the buffer zone. Statement "packet marking probability is very great $(\mathrm{H})$ " describe situations packet marking removed, discarded packets are not included in the buffer zone.

\subsection{Building fuzzy inference rules for FLRED}

Set fuzzy inference rules for FLRED are based on the set of values of the input variables $Q e(t), Q e(t-T)$ and output variables $p(t)$ has been built. The rules of the fuzzy controller is based on a formula (14). The system has two inputs, each input has seven linguistic variable value so be 49 fuzzy inference system. Rule form: if (deviation queue is $Q e(t))$ and (deviation queue before the $Q e(t-T))$, then the probability of packet marking removal is $p(t)$.

Table 5. Fuzzy rule system of FLRED

\begin{tabular}{|c|c|c|c|c|c|c|c|c|}
\hline \multirow{2}{*}{\multicolumn{2}{|c|}{$p(t)$}} & \multicolumn{7}{|c|}{$Q e(t-T)$} \\
\hline & & NB & NM & NS & ZE & PS & PM & PB \\
\hline \multirow{6}{*}{$Q e(t)$} & PB & VS & $\mathrm{T}$ & $\mathrm{Z}$ & $\mathrm{Z}$ & $\mathrm{Z}$ & $\mathrm{Z}$ & $\mathrm{Z}$ \\
\hline & PM & $\mathrm{S}$ & VS & $\mathrm{T}$ & Z & Z & Z & Z \\
\hline & PS & B & $\mathrm{S}$ & VS & $\mathrm{T}$ & Z & Z & Z \\
\hline & ZE & VB & B & $\mathrm{S}$ & VS & $\mathrm{T}$ & Z & $\mathrm{Z}$ \\
\hline & NS & $\mathrm{H}$ & VB & B & $\mathrm{S}$ & VS & $\mathrm{T}$ & $\mathrm{Z}$ \\
\hline & NM & $\mathrm{H}$ & $\mathrm{H}$ & VB & B & $\mathrm{S}$ & VS & $\mathrm{T}$ \\
\hline
\end{tabular}

\begin{tabular}{|l|l|l|l|l|l|l|l|l|}
\hline & NB & H & H & H & VB & B & S & VS \\
\hline
\end{tabular}

\subsection{The deductive surface of FLRED}

The FLRED inference surface is the shortest representation for its fuzzy controller. The real output $p(t)$ is an interpolation according to the two inputs Qe (t) and Qe (t-T). Reconciliation of nonlinear control surfaces and linguistic rules provides hints on the operation of the FLRED fuzzy control system. The method of using inference surfaces provides better probability of marking than other approaches, due to inference close to humans and available nonlinearity. Figure 7 illustrates the deductive surfaces of the law system of FLRED.

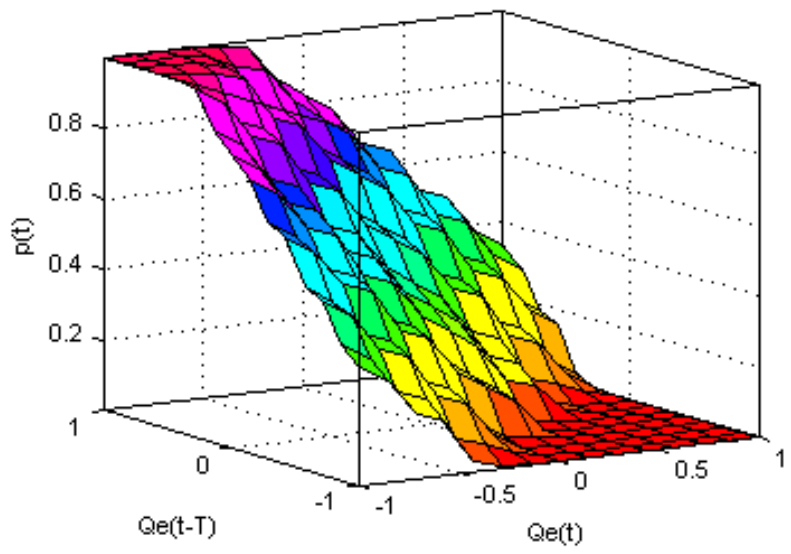

Figure 7. The deductive surface of the FLRED mechanism

From the fuzzy inference surface above, we can easily calculate the probability of marking in the equilibrium region, where the error on the queue length is almost zero. In areas where the queue length is below the reference level, the probability of marking the packet is considered to be zero. In contrast, the rules are active by dramatically increasing packet markup in the queue length area beyond the reference level, where congestion begins to be established.

\subsection{Illustrate calculating output of FLRED}

In order to have a better view of the operation of the fuzzy system of FLRED, analyze the illustrative example of the operation of its fuzzy controller through Figure 8. Assume that at the end of two consecutive sample intervals, the instant queue length errors at two points (t) and (tT) relative to the reference queue length are: $\mathrm{Qe}(\mathrm{t})=-0.5, \mathrm{Qe}(\mathrm{tT})=0.5$. At the previous time (t-T), the queue length was below the reference level. At the instant ( $\mathrm{t}$ ), the queue length is above the reference level. Thus, the usage of queue length tends to go far beyond the reference level of TQL. The current deviation of the queue length Qe $(t)$ is a member of the fuzzy set "NM" and "NS" with dependent values of 0.5 for each fuzzy set. The flux error before Qe (t-T) is a member of the fuzzy set "PS" and "PM" with dependent values of 0.5 and 0.5 , respectively. The dependency value of any other fuzzy set of the two input variables is zero. For the numerical values of the input variables used in this example, only rules $12,13,19$ and 20 of the deductive system contribute to the calculation of the output. The sum of all four rules and the use of the 
central defocusing method will give the output value the packet marker / discard probability of $\mathrm{p}(\mathrm{t})=0.832$.

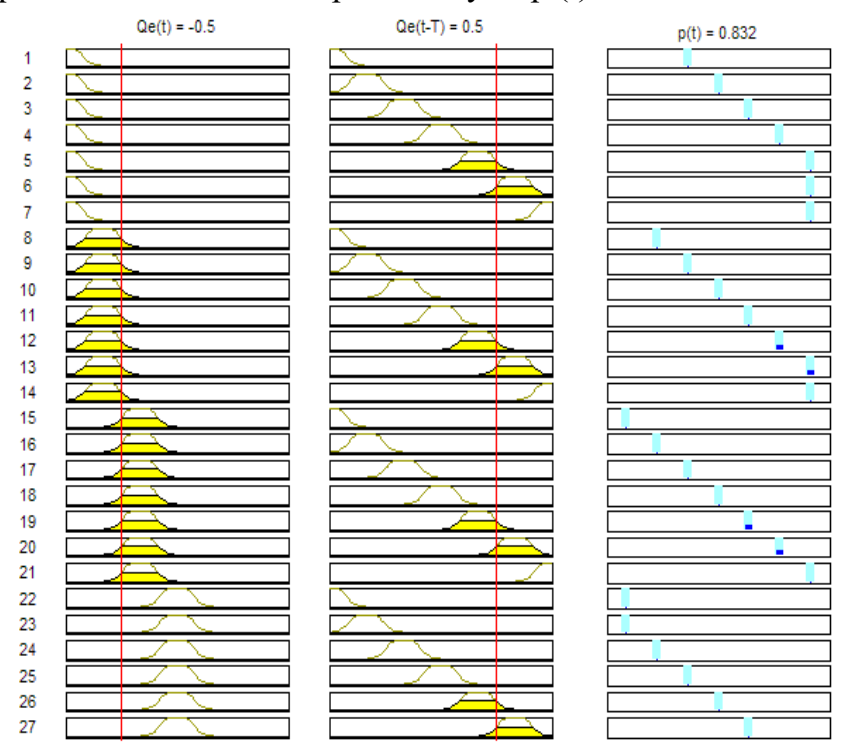

Figure 8. Illustrate calculating output of FLRED

\section{Improve REM mechanisms by AFC}

REM mechanism is typical mechanism for AQM mechanisms based on queue length and flow rate to. Therefore, improvement in the AQM mechanism by AFC adaptive fuzzy control, we also choose to improve REM represents AQM mechanisms based on queue length and flow rate to. AFC adaptive fuzzy controller model for the mechanism FLREM builds on AFC general model for improving AQM mechanisms is presented in Section 4.1. Based on this model, the fuzzy controller's AFC FLREM built as follows.

\subsection{Fuzzy input variables for FLREM}

AFC for FLREM also based on variable rates $\left(P_{r}\right)$ measure of congestion as REM algorithm. Therefore, we use two inputs, one for the sample at the current time $\operatorname{Pr}(t)$ and a time to form in the previous cycle $\operatorname{Pr}(t-T)$. Based on these two input values, the fuzzy controller determines the value of the packet marking probability represents the output of the fuzzy system.

To improve the accuracy we increase the number of membership functions for input and output of the fuzzy system is 9 , this will make some inference rules become 81 rules. The downside of the calculation of the price mechanism is the magnitude of the price REM always positive, this is not the actual queue length and flow rate to be much smaller than the reference queue length and bandwidth transmission.

The value of the selected input language to denote the case corresponding to two consecutive times and fuzzy membership functions by a bell-shaped domain nine values: $P_{r}(t)=\{N H, N B, N M, N S, Z E, P S, P M, P B, P H\} \quad$ and $P_{r}(t-T)=\{N H, N B, N M, N S, Z E, P S, P M, P B, P H\} . \quad$ In particular: Negative Huge $(\mathrm{NH})$, Negative Big (NB), Negative Medium (NM), Negative Small (NS), Zero (ZE), Positive Small (PS), Positive Medium (PM), Positive Big (PB), Positive Huge (PH).

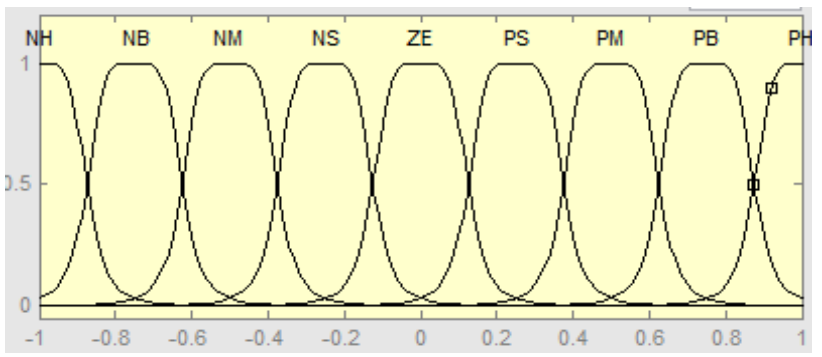

Figure 9. Membership functions of the input variables FLREM

\subsection{Fuzzy output variables for FLREM}

The value of language in the output variable is the singleton value represents the probability nine ascending remove packages from the interval $[0,1]$, is defined as follows:

$$
\begin{aligned}
& p(t)=\{Z, T, V S, M S, S, B, M B, V B, H\} \\
& p(t)=\{0,0.125,0.25,0.375,0.5,0.635,0.75,0.875,1\}
\end{aligned}
$$

In particular, Zero (Z), Tiny (T), Very Small (VS), Medium Small (MS), Small (S), Big (B), Medium Big (MB), Very Big (VB), and Huge (H).

\subsection{Building inference rules for FLREM}

Inference system for fuzzy controller consists of a set of If then rules according to general rules in the form of formula (14). To facilitate the build fuzzy rules, the index for the domain associated values of the input linguistic variables $e(t), e(t-T)$ is $\{N H, N B, N M, N S, Z E, P S, P M, P B, P H\}=$ $\left\{\mu(e)_{-4}, \mu(e)_{-3}, \mu(e)_{-2}, \mu(e)_{-1}, \mu(e)_{0}, \mu(e)_{1}, \mu(e)_{2}, \mu(e)_{3}, \mu(e)_{4}\right\}$ , the output linguistic variable packet marking probability $p(t)$ is $\{H, V B, M B, B, S, M S, V S, T, Z\}=$

$\left\{\mu(p)_{-4}, \mu(p)_{-3}, \mu(p)_{-2}, \mu(p)_{-1}, \mu(p)_{0}, \mu(p)_{1}, \mu(p)_{2}, \mu(p)_{3}, \mu(p)_{4}\right\}$. Fuzzy system is built according to the following general form:

If $e(t)=\mu(e(t))_{i}$ và $e(t-T)=\mu(e(t-T))_{j} \quad$ Then $p(t)=\mu(p(t))_{k}$

In particular: $i, j=\overline{-4,4}$, and $k=f(i, j)=i+j$, if $i+$ $j>4$ then $f(i, j)=4$ and if $i+j<-4$ then $f(i, j)=-4$.

Based on generalized fuzzy systems, building rules in the following Table 6:

Table 6 . Index systems of fuzzy rules for FLREM

\begin{tabular}{|c|c|c|c|c|c|c|c|c|c|c|}
\hline \multirow{2}{*}{$p(t)$} & \multicolumn{7}{|c|}{$\operatorname{Pr}(t-T)$} \\
\cline { 2 - 11 } & -4 & -3 & -2 & -1 & 0 & 1 & 2 & 3 & 4 \\
\hline \multirow{6}{*}{} & 4 & 0 & 1 & 2 & 3 & 4 & 4 & 4 & 4 & 4 \\
\cline { 2 - 11 } & 3 & -1 & 0 & 1 & 2 & 3 & 4 & 4 & 4 & 4 \\
\cline { 2 - 10 } & 2 & -2 & -1 & 0 & 1 & 2 & 3 & 4 & 4 & 4 \\
\cline { 2 - 11 } & 1 & -3 & -2 & -1 & 0 & 1 & 2 & 3 & 4 & 4 \\
\cline { 2 - 11 } & 0 & -4 & -3 & -2 & -1 & 0 & 1 & 2 & 3 & 4 \\
\cline { 2 - 11 } & -1 & -4 & -4 & -3 & -2 & -1 & 0 & 1 & 2 & 3 \\
\cline { 2 - 11 } & -2 & -4 & -4 & -4 & -3 & -2 & -1 & 0 & 1 & 2 \\
\cline { 2 - 11 } & -3 & -4 & -4 & -4 & -4 & -3 & -2 & -1 & 0 & 1 \\
\hline
\end{tabular}




\begin{tabular}{ll|l|l|l|l|l|l|l|l|l|}
-4 & -4 & -4 & -4 & -4 & -4 & -3 & -2 & -1 & 0 \\
\hline
\end{tabular}

Based on the domain index values of the linguistic variables for input and output in Table 4, build system for FLREM rules as Table 7. System rules for the fuzzy controller is made up of the control rule of the form:

Table 7. Fuzzy rule system of FLREM

\begin{tabular}{|c|c|c|c|c|c|c|c|c|c|c|}
\hline \multirow{2}{*}{$\mathrm{p}(\mathrm{t})$} & \multicolumn{10}{|c|}{$\mathrm{e}(\mathrm{t}-\mathrm{T})$} \\
\cline { 2 - 12 } & NH & NB & NM & NS & ZE & PS & PM & PB & PH \\
\hline \multirow{5}{*}{ e(t) } & PH & S & MS & VS & T & Z & Z & Z & Z & Z \\
\cline { 2 - 11 } & PB & B & S & MS & VS & T & Z & Z & Z & Z \\
\cline { 2 - 11 } & PM & MB & B & S & MS & VS & T & Z & Z & Z \\
\cline { 2 - 11 } & PS & VB & MB & B & S & MS & VS & T & Z & Z \\
\cline { 2 - 11 } & NS & H & H & VB & MB & B & S & MS & VS & T \\
\cline { 2 - 11 } & NM & H & H & H & VB & MB & B & S & MS & VS \\
\cline { 2 - 11 } & NB & H & H & H & H & VB & MB & B & S & MS \\
\cline { 2 - 11 } & NH & H & H & H & H & H & VB & MB & B & S \\
\hline
\end{tabular}

\subsection{The deductive surface of FLREM}

The FLREM deductive surface shown in Figure 10 represents the most abridged representation of its fuzzy controller. The real output $\mathrm{p}(\mathrm{t})$ is an interpolation according to the two inputs $\operatorname{Pr}(\mathrm{t})$ and $\operatorname{Pr}(\mathrm{t}-\mathrm{T})$. Reconciliation of the nonlinear control surface and the language rules shown in Table 7, will provide suggestions on the operation of the fuzzy control system of the FLREM mechanism.

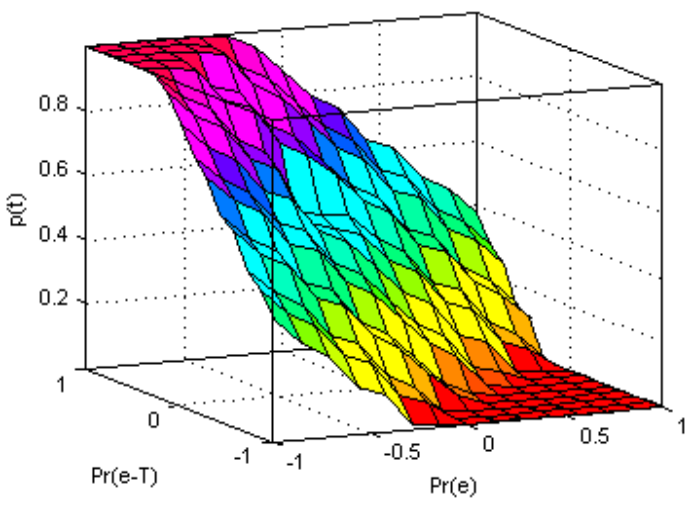

Figure 10. The deductive surface of the FLREM mechanism

\section{Simulation and evaluation}

Simulation AQM mechanisms are implemented in NS2 software, this software is the most widely used, the research community is trusted and recognized. To objectively evaluate the AQM mechanism, the mechanisms is installed on the same network environment simulation process and are evaluated based on the following criteria: control the queue length and the ability to respond to network changes.

To evaluate, compare the performance of AQM mechanisms must be installed simulation mechanisms in a network model, similar to other network environments, such as the number of nodes, network diagrams, of the connections, applications, bandwidth and latency on each connection. At the same time all the simulation results for AQM mechanisms have processes are described in section 7.1.

The performance of AQM mechanisms will be analyzed and evaluated based on the same general criteria system, is presented in Section 7.2, such as transmission efficiency, packet loss rate in knots bottleneck, the average queuing delay and delay variation.

\subsection{Evaluation of stability of FLRED and FLREM mechanisms}

For the purpose of evaluating the effectiveness of the AQM mechanism using fuzzy controllers AFC, we choose to compare with the original AQM mechanism represents the group (such as RED, REM) and AQM mechanisms using fuzzy control (such as FEM and FUZZEM) for representing primitive. Therefore, the network model to simulate the mechanism in our model was used to simulate the RED, REM, FEM, FUZREM mechanisms. This model is shown in Figure 11. [8][16][25][30].

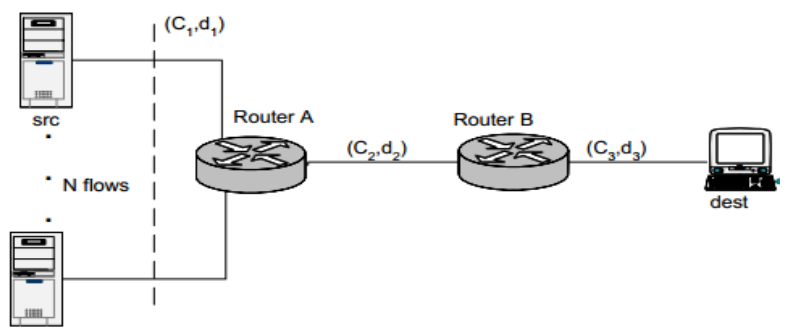

Figure 11. The network model to simulate the AQM

In the model, using the communication protocol TCP / NewReno with congestion window is 240 packets, each packet size of 1000 bytes. Buffer size of all queues is 500 packets. Queue of the bottleneck link is placed between the router-router-A and $\mathrm{B}$, respectively installing AQM algorithms queue manager at bottlenecks, all other links placed queue management mechanism passive. Reference queue length for the mechanism to be set to 200 packets $(40 \%$ buffer size). Sampling distance is 0.1 seconds, the time taken for the article simulation mechanisms is 100 seconds.

\section{Control the queue of $A Q M$ mechanisms}

The objective of the first simulation scenario is evaluated control queue length of AQM mechanisms in real time. For this purpose, set the number of connections in the source $\mathrm{N}=$ 60 , the bandwidth and latency of the connection is: $(\mathrm{C} 1, \mathrm{~d} 1)=$ $(15 \mathrm{Mbps}, 40 \mathrm{~ms}),(\mathrm{C} 2, \mathrm{~d} 2)=(15 \mathrm{Mbps}, 5 \mathrm{~ms})$, and $(\mathrm{C} 3, \mathrm{D} 3)=$ (30Mbps, 5ms). For the RED queue, simulation set reference queue length 200 package and set two thresholds $\min _{t h}$ and max $_{\text {th }}$ corresponding $30 \%$ and $70 \%$ of the queue length. Congestion notification Mechanism of RED has been made possible by actively setting $\max _{p}$ is 1 . Queue reference for FLRED mechanism is set in turn is 100 packages, 200 packages and 300 packages.

The simulation results of the RED, FEM, and mechanisms 
is shown in Figure 12, shows that the mechanism of improved mechanisms of RED mechanism using fuzzy control as FEM and FLRED always have the ability to control every wait relatively stable over traditional RED mechanism. This may be due to the fuzzy controller of the mechanisms controlling queues based on the queue reference. In addition, FLRED mechanisms have a smaller amplitude than the FEM mechanism, the mechanism by FLRED get fuzzy controller according to a bell-shaped smooth and adjust the value in the packet marking probability output of the fuzzy controller according to the variation of the network
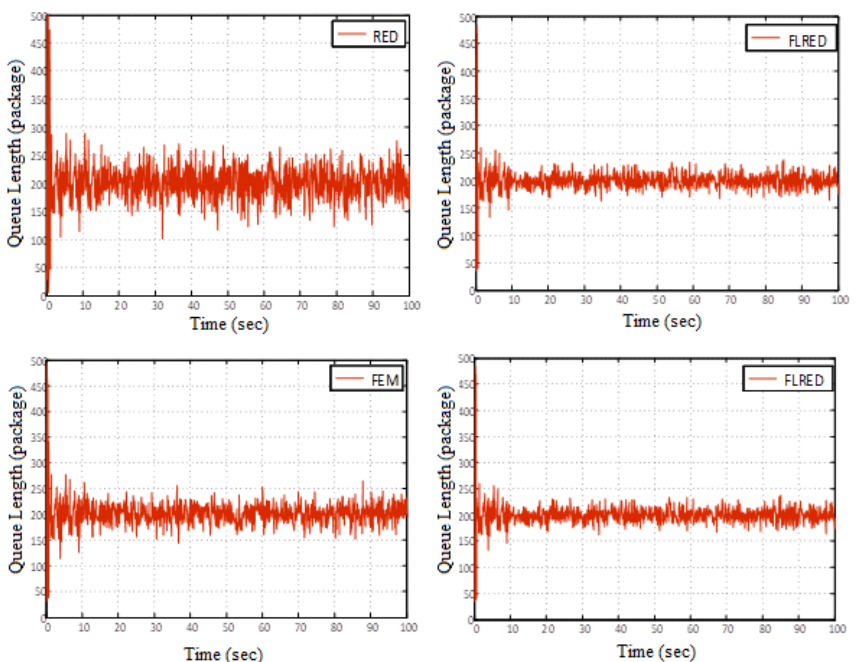

Figure 12. Control the queue of RED, FEM and FLRED

When the simulation REM mechanism and the mechanisms to improve REM have used fuzzy controller (FUZREM, FLREM), results in Figure 13, shows that the mechanism controlling REM queue is unstable, whereas the FUZREM and FLREM mechanisms keep the immediate queue length at network nodes is relatively stable. However, due FLREM nine bell membership function as fuzzy input values so be increased precision makes it possible queue stability better than FUZREM.
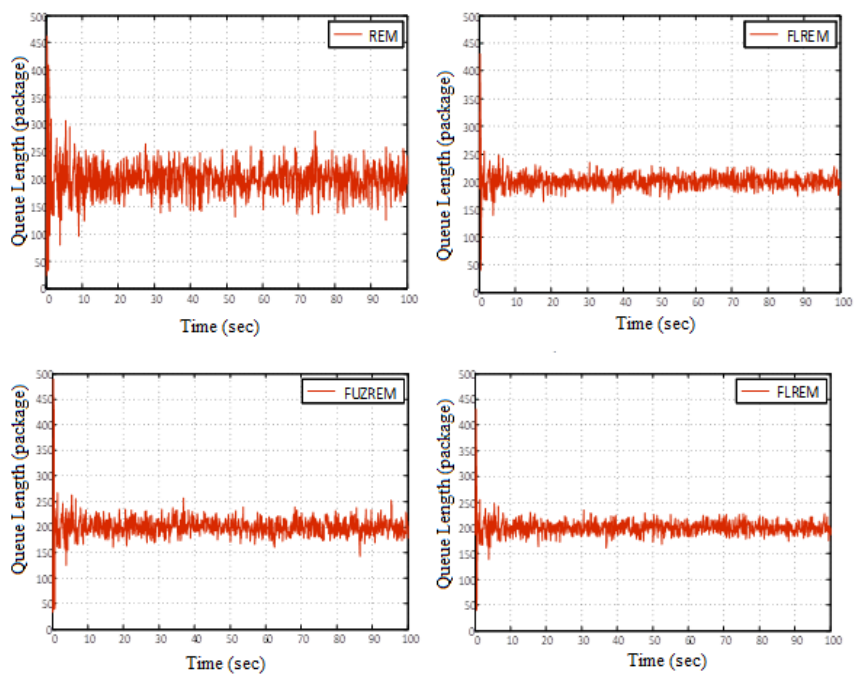

Figure 13. Control the queue of REM, FUZREM and FLREM

\section{Ability to respond of $A Q M$ mechanisms}

Simulation test the ability to respond of AQM mechanisms when the characteristics of of TCP change through changing the number of connections from 60 to 100 and the access time to the network by stopping the flow in half at time $t=40$ seconds and recover in time $\mathrm{t}=70$ seconds.

Figure 14 and Figure 15 shows, the more reduced the number of connections in the 40th second, the immediate queue length of the mechanism immediately dropped. When increasing the number of TCP flows to 100 , the number of packets to the queue and thus increase efficiency queue will increase, leading to delay and delay variation in the queue increases. However, the FEM and FLRED mechanisms, FUZREM and FLREM maintain the queue length fluctuated queue reference value.
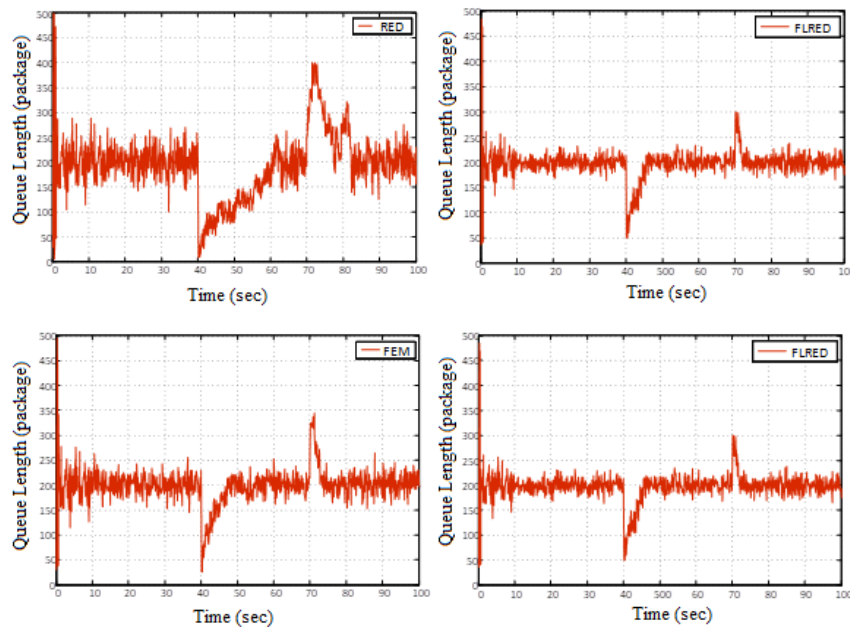

Figure 14. Ability to respond of RED, FEM and FLRED

Ability to respond the queue management mechanism based on queue length is shown in Figure 14, again shows the adaptation of FEM and FLRED mechanisms when using fuzzy control and the ability to restoration of the status queue FLRED mechanism high when fuzzy system with bell.

Similarly, Figure 15 represents ability to respond the queue management mechanism based on queue length and flow rate to, FLREM have a shorter recovery time than FUZREM.
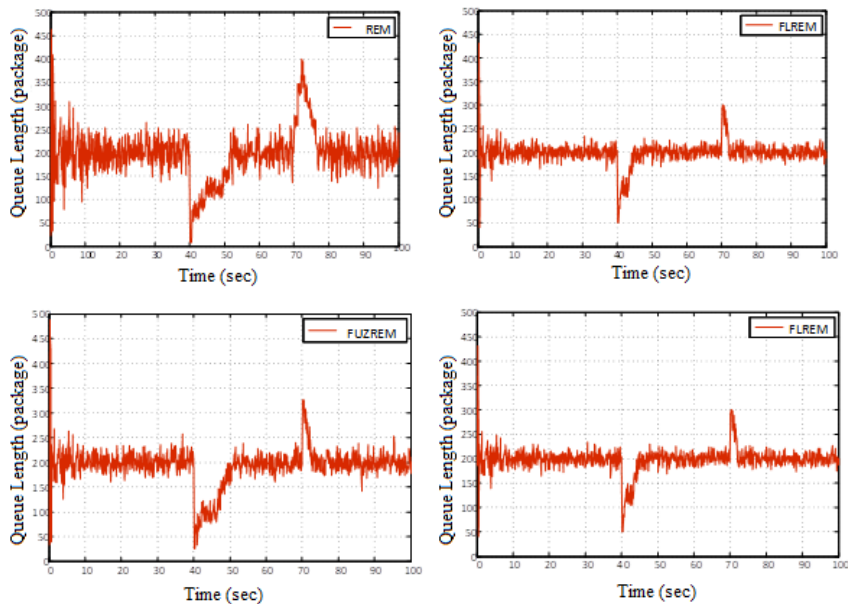

Figure 15. Ability to respond of REM, FUZREM, FLREM

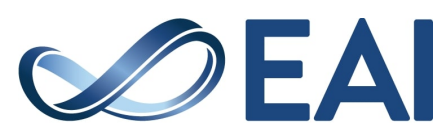




\subsection{Performance evaluation of FLRED and FLREM mechanisms}

To evaluate the effectiveness of AQM mechanisms when using the fuzzy controller, we use the general network model in Figure 16 to extend the simulation process for the sending, receiving and multithreading cases. queue. The general model is a classic script that has been widely used in scientists' research projects, with different types of flux sharing a bottleneck location [12] [36].

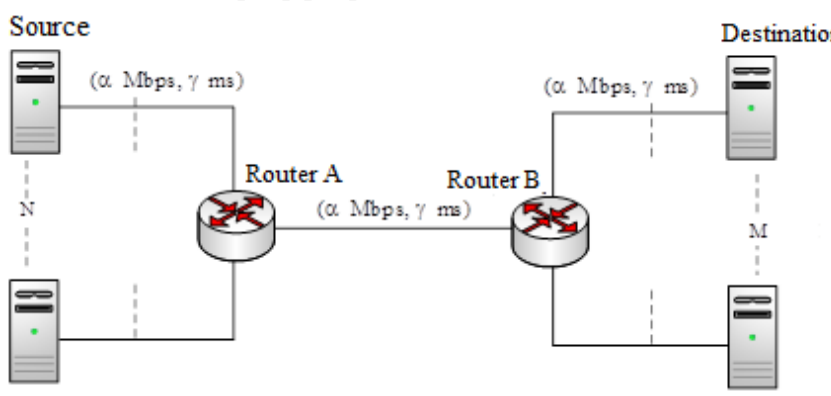

Figure 16. Network model simulating multiple receivers

In the simulation, the use of TCP streams $\mathrm{N}$ varies from 10 to 300 , the queue length at the bottleneck varies from 100 to 1000. The lines from the sending machines to the bottleneck and from the bottleneck to the The receiver has a bandwidth of $15 \mathrm{Mbps}$ and a delay of $20 \mathrm{~ms}$ varies according to specific models. Bottlenecks in a scenario where the bandwidth is 15 Mbps and the delay is $20 \mathrm{~ms}$. The network bottleneck is in turn installed the algorithm of the mechanism for evaluation. The buffer size at $\mathrm{Q}$ bottleneck varies from case to case to show the dynamism of the network in the simulation model.

To evaluate the performance of mechanisms in the context of network fluctuations, we construct two main scenarios: The first scenario, for the queue length at the bottleneck to vary from 100 to 1000 while the number of connection flows constant (60 connections); The second scenario, for the load changes (the number of threads varies) from 10 to 300 while the queue length at the bottleneck remains constant (500 packets). Set up TCP / NewReno communication protocol with congestion window of 240 packets, the size of each packet is 1000 Bytes. The buffer size of all queues is 500 packets. The reference queue length for the mechanisms is set to 200 packets ( $40 \%$ of buffer size).

\section{Evaluate packet loss rate of FLRED and FLREM mechanisms}

Figure 17 shows that when the queue queue size increases, the packet loss rate of the mechanisms decreases and as the number of flows connected to the router increases, the rate of packet loss increases. This is consistent with the change of the network environment, as network resources increase (queue length increases), packet loss decreases and as load increases (the number of connection flows increases), packet loss increases accordingly.
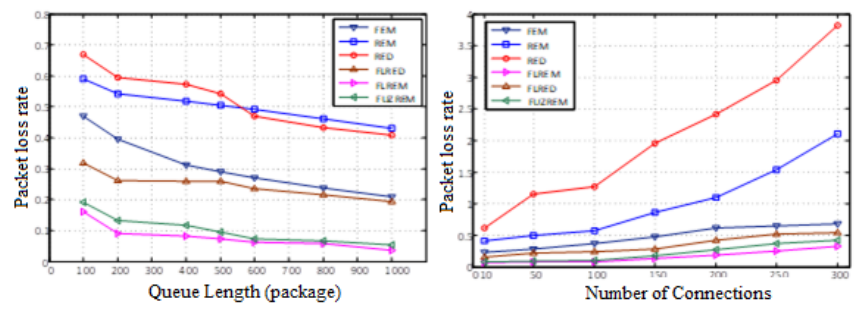

Figure 17. Packet loss rate of AQM mechanisms

On the graph, the lines represent the packet loss rate of the mechanisms with partitioning into three groups. The top group belongs to the mechanisms that do not use fuzzy control (RED, REM), the group immediately below is the RED improvement mechanisms (FEM, FLRED), the bottom group is of the improved mechanisms. REM (FUZREM, FLREM). When improved on the same mechanism (RED, REM), the mechanism using adaptive fuzzy control (FLRED, FLREM) has a lower packet loss rate than that of traditional fuzzy control (FEM, FUZREM). This result is consistent with the operating principle of traditional fuzzy controllers and adaptive fuzzy controllers. With adaptive fuzzy control, in addition to the Sugeno fuzzy system, there is also an adaptive mechanism to adjust the probability of packet marking in accordance with network changes.

\section{Evaluate throughput of FLRED and FLREM mechanisms}

The graph in Figure 18 shows the data on transmission usage of the mechanisms. The ability to take advantage of the transmission of the mechanisms increases as queue size and load increase. The mechanism uses fuzzy control always use level greater than $90 \%$ transmission, higher than the use of transmission lines of traditional mechanisms. This is consistent with the control of active queue management mechanisms, when using fuzzy control, the processing is simpler and more efficient than mechanisms that do not use fuzzy control.
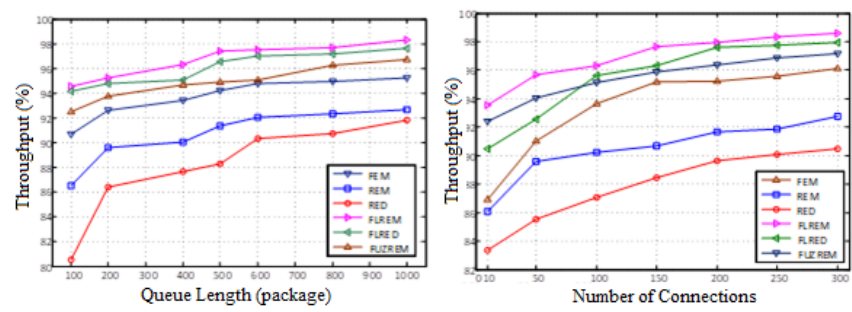

Figure 18. Throughput of AQM mechanisms

In all cases, the RED mechanism always has the lowest throughput and the FLREM mechanism always has the highest throughput. This result is due to FLREM mechanism which converges criteria when processing packets to the network node. In addition to the inheritance of the REM mechanism when considering the effect of queue length and load, the FLREM mechanism is also improved by an adaptive fuzzy controller. While RED uses only the length of the queue to determine probability. 


\section{Conclusions}

Operational mechanisms for congestion control at the network nodes are essential. Therefore, intelligent computing tools such as fuzzy controls should be included in the mechanisms to make network nodes more efficient to improve service quality and network performance. We have improved the queue management mechanism by introducing adaptive fuzzy controller - AFC into the process of active queue management of the network by using Sugeno fuzzy system and the differential operation to adjust output and input variables of the fuzzy controller. Based on the theoretical model, we deploy to install the simulation, develop FLRED and FLREM innovative mechanisms. The simulation results show that the mechanisms are innovated by improving the adaptive fuzzy controller AFC to control the queue better than traditional mechanisms and mechanisms previously using fuzzy logic. Thus, the adaptive fuzzy controller supports to enhance the management performance of the active queue management mechanisms

\section{References}

[1] Alexander Afanasyev, Neil Tilley, Peter Reiher, and Leonard Kleinrock (2010), "Host-to-Host Congestion Control for TCP", IEEE Communications Surveys \& Tutorials, Accepted For Publication, pp. 1-39.

[2] Alshalaa A. Shleeg, Issmail M. Ellabib (2013), "Comparison of Mamdani and Sugeno Fuzzy Interference Systems for the Breast Cancer Risk", International Journal of Computer, Control, Quantum and Information Engineering, Vol. 7, No. 10, pp. 695699.

[3] Arash Danal and Ahmad Malekloo (2010), "Performance Comparison between Active and Passive Queue Management", JCSI International Journal of Computer Science Issues, Vol. 7, Issue 3, No. 5, pp. 13-17.

[4] Arshdeep Kaur, Amrit Kaur (2012), "Comparison of MamdaniType and Sugeno-Type Fuzzy Inference Systems for Air Conditioning System", International Journal of Soft Computing and Engineering (IJSCE), Vol. 2, Issue-2, pp. 322-325.

[5] Athuraliya S., Lapsley D. E., Low S. H. (2001), "Random early marking for Internet congestion control”. IEEE/ACM Transactions on Networking, Vol. 15, No. 3, pp. 48-53.

[6] Bartek Peter Wydrowski (2003), Techniques in Internet Congestion Control, Electrical and Electronic Engineering Department The University of Melbourne.

[7] C. Chryostomou (2006), "Fuzzy logic based AQM congestion control in TCP/IP networkfor Quality of Service Provisioning ", Department of Computer of Science, University of Cyprus.

[8] C. Chryostomou, A. Pitsillides, G. Hadjipollas and others (2007), "Fuzzy Logic Congrestion Control in TCP/IP Best-Effort Networks". University of Cyprus, Monash University Melbourne, Australia, pp. 2-5.

[9] C. V. Hollot, V. Misra, D. Towsley, and W. Gong (2002), "Analysis and design of controllers for AQM routers supporting TCP flows", IEEE Trans. on Automat. Control, No. 47, pp. 945959.

[10] Chhabra Kiran, Manali Kshirsagar, A. S. Zadgaonkar (2013), "Effective Congestion Indication for Performance Improvement of Random Early Detection", International Journal of Innovative Technology and Exploring Engineering (IJTTEE), Vol. 3, pp. 3538.
[11] Dong Lin and Robert Morris (1997), "Dynamics of random early detection", In SIGCOMM 97: Proceedings of the ACM SIGCOMM "97 conference on Applications, technologies, architectures, and protocols for computer communication, New York, NY, USA, ACM Press., pp.127-137.

[12] E. Park, H. Lim, K. Park, and C. Choi (2004), "Analysis and design of the virtual rate control algorithm for stabilizing queues in TCP networks", Computer Networks, Vol. 44, No. 1, pp. 1741.

[13] Floyd, S., Gummadi, R., \& Shenker, S. (2001), "Adaptive RED: An Algorithm for Increasing the Robustness of RED Active Queue Management", Technical report, ICSI, AT\&T Center for Internet Research at ICSI, pp.1-12.

[14] G.F.Ali Ahammed, Reshma Banu (2010), "Analyzing the Performance of Active Queue Management Algorithms", International journal of Computer Networks \& Communications (IJCNC), Vol.2, No.2, pp. 1-12.

[15] G.Thiruchelvil and J.Raja (2008), "A Survey On Active Queue Management Mechanisms", IJCSNS International Journal of Computer Science and Network Security, VOL.8 No.12, 130-145.

[16] Hao Kun, Wang Beibei, Luo Yongmei (2013), "Study of AQM Congestion Control Algorithm Based on Control Theory", Journal of Convergence Information Technology(JCIT), Vol. 8, No. 8, pp. 600-607.

[17] I. K. Tabash, M. A. A. Mamun, and A. Negi (2010), “A Fuzzy Logic Based Network Congestion Control Using Active Queue Management Techniques", J. Sci. Res. 2 (2), pp. 273-284.

[18] J. Sun, M. Zukerman, and M. Palaniswami (2007), "Stabilizing RED using a fuzzy controller," in Proceedings of the IEEE International Conference on Communications (ICC '07), pp. 266-271.

[19] Jasem, H.N., Z.A. Zukarnain, M. Othman and S. Subramaniam (2011), "Efficiency and Fairness of New-Additive Increase Multiplicative Decrease Congestion Avoidance And Control Algorithm", J. Applied Sci, No. 11, pp. 438-449.

[20] Jyh Shing Roger Jang, Chuen Tsai Sun, Eiji Mizutani (2002), Neuro fuzzy and Soft Computing, Prientice Hall International, Inc.

[21] Kaur, Gurmeet; Singh, M. L (2009), "A Survey of Recent Advances in Fuzzy Logic in Communication Systems", International Journal of Applied Engineering Research, Vol. 4, Issue 2, p139 -145.

[22] Kevin Fall and Kannan (2010), The NS Manual, A Collaboration between researchers at UC Berkeley, LBL, USC/ISI, and Xerox PARC.

[23] M. H. Yaghmaee, M. B. Menhaj, H. Amintoosi (2005), “A Fuzzy Extension to the BLUE Active Queue Management Algorithm", Journal of Iranian Association of Electrical and Electronics Engineers, Vol.1, No.3, pp. 3-14.

[24] M. Moghaddam (2010), "A fuzzy active queue management mechanism for Internet congestion control", in Proceedings of the 2010 Third International Workshop on Advanced Computational Intelligence (IWACI), pp. 203-208.

[25] Michael Welzl (2005), Network Congestion Control Managing Internet Traffic, John Wiley \& Sons Ltd.

[26] Minseok Kwon and Sonia Fahmy (2010), “A Comparison of Load-based and Queue-based Active Queue Managgement Algorithms", Dept. Of computer Science, Purdue University, West Lafayette, in 47906-1398, USA, pp.1-12.

[27] Mohammed Z. Al-Faiz, Shahad A. Sadeq (2012), "Particle Swarm Optimization Based Fuzzy-Neural Like PID Controller for TCP/AQM Router", Intelligent Control and Automation, No. 3, pp. 71-77.

[28] P. Singh and S. Gupta (2011), "Variable length virtual output queue based fuzzy congestion control at routers", in Proceedings 
of the IEEE 3rd International Conference on Communication Software and Networks (ICCSN), pp. 29-33.

[29] Richelle Adams (2013), "Active Queue Management: A Survey", IEEE communications surveys \& tutorials, Vol. 15, No. 3, pp. 1425-1476.

[30] S. Athuraliya, S. Low, V. Li, and Q. Yin (2001), "REM: Active Queue Management", IEEE Network, Vol. 15, No. 3, pp. 48-53.

[31] S. H. Low, F. Paganini, and J. C. Doyle (2002). Internet congestion control. IEEE Control Systems Magazine, Feb 2002

[32] S. Zargar, M. Yaghmaee, and A. Fard (2006), "Fuzzy Proactive Queue Management Technique", in Proceedings of the 2006 Annual IEEE India Conference, pp. 1-6.

[33] Sanjeewa Athuraliya, Steven H. Low, Victor H. Li and Qinghe Yin (2001), "REM: Active Queue Management", IEEE Network, pp. 48-53.

[34] Shilpa N. Ingoley, Madhu Nashipudi (2012), “A Review Fuzzy Logic in Congestion Control of Computer Network", Proceedings published in International Journal of Computer Applications ${ }^{\circledR}(0975$ - 8887), pp. 1-6.
[35] Tanvi B. Harmalkar, H. G. Virani (2013), "Neural Network Active Queue Management For Congestion Control", Tanvi Harmalkar, IJPRET, 2013, Vol. 1, pp. 572-580.

[36] V. Santhi, A. M. Natarajan (2011), "Active Queue Management Algorithm for TCP Networks Congestion Control", European Journal of Scientific Research, ISSN 1450-216X, Vol.54 No.2, pp. 245-257

[37] W. Feng, K. Shin, D. Kandlur, and D. Saha (2002), "The BLUE Active Queue Management Algorithms", IEEE/ACM Transactions on Networking, Vol. 10, No. 4, pp. 513-528.

[38] X. Changbiao and L. Fengfeng (2008), "A Congestion Control Algorithm of Fuzzy Control in Routers", in Proceedings of the Fourth International Conference on Wireless Communications, Networking and Mobile Computing (WiCOM '08), October 2008, pp. 1-4.

[39] Y. Qiao and H. Xiaojuan (2010), “A new PID controller for AQM based on neural network", in Proceedings of the 2010 IEEE International Conference on Intelligent Computing and Intelligent Systems (ICIS), Vol. 1, pp. 804-808. 